\title{
What does best practice care for musculoskeletal pain look like? Eleven consistent recommendations from high-quality clinical practice guidelines: systematic review
}

\author{
Ivan Lin (1) , ${ }^{1}$ Louise Wiles, ${ }^{2}$ Rob Waller, ${ }^{3}$ Roger Goucke, ${ }^{4}$ Yusuf Nagree, ${ }^{5,6}$ \\ Michael Gibberd, ${ }^{7}$ Leon Straker $10,{ }^{8}$ Chris G Maher, ${ }^{9}$ Peter P B O'Sullivan ${ }^{10}$
}

- Additional material is published online only. To view please visit the journal online (http://dx.doi.org/10.1136/ bjsports-2018-099878).

For numbered affiliations see end of article.

\section{Correspondence to} Dr Ivan Lin, WA Centre for Rural Health, University of Western Australia, Western Australia, 6531, Australia; ivan.lin@uwa.edu.au

Accepted 8 February 2019 Published Online First 2 March 2019

\begin{abstract}
Objectives To identify common recommendations for high-quality care for the most common musculoskeletal (MSK) pain sites encountered by clinicians in emergency and primary care (spinal (lumbar, thoracic and cervical), hip/knee (including osteoarthritis [OA] and shoulder) from contemporary, high-quality clinical practice guidelines (CPGs).
\end{abstract}

Design Systematic review, critical appraisal and narrative synthesis of MSK pain CPG recommendations. Eligibility criteria Included MSK pain CPGs were written in English, rated as high quality, published from 2011, focused on adults and described development processes. Excluded CPGs were for: traumatic MSK pain, single modalities (eg, surgery), traditional healing/medicine, specific disease processes (eg, inflammatory arthropathies) or those that required payment.

Data sources Four scientific databases (MEDLINE Embase, CINAHL and Physiotherapy Evidence Database) and four guideline repositories.

Results 6232 records were identified, 44 CPGs were appraised and 11 were rated as high quality (low back pain: 4, OA: 4, neck: 2 and shoulder: 1). We identified 11 recommendations for MSK pain care: ensure care is patient centred, screen for red flag conditions, assess psychosocial factors, use imaging selectively, undertake a physical examination, monitor patient progress, provide education/information, address physical activity/exercise, use manual therapy only as an adjunct to other treatments, offer highquality non-surgical care prior to surgery and try to keep patients at work.

Conclusion These 11 recommendations guide healthcare consumers, clinicians, researchers and policy makers to manage MSK pain. This should improve the quality of care of MSK pain.

\section{BACKGROUND}

Musculoskeletal (MSK) pain conditions are the biggest cause of disability internationally ${ }^{1}$ and a major societal burden. One contributor to this burden, which is becoming increasingly recognised, is poor quality healthcare. ${ }^{2}$ Common healthcare problems for MSK pain are summarised in box 1 and include overuse of radiological imaging, surgery and opioids and a failure to provide patients with education and advice.

\section{What is already known}

- There is an urgent need to improve quality of care for musculoskeletal (MSK) pain conditions. This is a priority for clinicians, health care services, researchers and policy makers.

- MSK pain in different body areas share similar features, and it may be possible to identify consistent overarching recommendations for assessment and management. Identifying common recommendations could be a useful way to improve care quality.

\section{What are the new findings}

- We identified 11 consistent recommendations for MSK pain from high-quality clinical practice guidelines.

- These could be used to improve MSK pain care by assessing care quality, for example, through audit, guide clinical decision making and identify clinician education needs.

Evidence-to-practice gaps such as these are problematic because they waste healthcare resources and prevent patients from receiving appropriate care.

One of the 'foundations of efforts to improve healthcare, ${ }^{3}$ and to reduce evidence-to-practice gaps, are clinical practice guidelines (CPGs). CPGs are 'statements that include recommendations intended to optimise patient care that are informed by a systematic review of evidence and an assessment of the benefits and harms of alternative care options'. ${ }^{4} \mathrm{CPGs}$ aim to improve care in a number of ways including: guiding clinical and patient decision making, acting as a standard of care, contributing to the development of clinical decision-making aids, informing all stakeholders about what constitutes best practice and guiding allocation of healthcare resources. ${ }^{4}$

Care that is more concordant with CPG recommendations results in better patient outcomes and lower costs. ${ }^{5}{ }^{6}$ However, CPGs have been criticised because clinicians have difficulty using them. Issues include: a multitude of CPGs for the one condition or when they are voluminous documents that are not user-friendly, ${ }^{7}$ shortcomings 
Box 1 Summary of common problems in musculoskeletal (MSK) pain care

- Overuse of imaging: between $25 \%$ and $42 \%$ of patients with low back pain (LBP) undergo imaging ${ }^{349}$ even though its routine use is discouraged and associated with harms. Sixtynine per cent of general practitioners would refer patients for radiography at first presentation of rotator cuff tendinopathy and $82 \%$ would refer for ultrasound, ${ }^{35}$ despite findings demonstrating a poor relationship of imaging findings with symptoms. ${ }^{50}$

- Overuse of surgery: knee arthroscopy for knee osteoarthritis is not recommended, yet its rate of use in the general US population increased from $3 \%$ to $4 \%$ from 2006 to $2010 .{ }^{51}$ The rates of shoulder subacromial decompression and rotator cuff repair ${ }^{52}$ have increased markedly even though surgical outcomes are comparable with exercise-based rehabilitation ${ }^{53}$ or sham surgery. ${ }^{54}$

- Overuse of opioids: the efficacy of opioids for MSK pain management is questionable for both chronic ${ }^{55}$ and acute MSK pain conditions. ${ }^{42}$ The early use of opioids has been associated with poorer outcomes in LBP care ${ }^{56}$ Although limiting the use of opioids is recommended, ${ }^{44}$ there is increasing use and an 'epidemic' of prescription opioidrelated harms. $^{57}$

- Failure to provide education and advice: these are cornerstones of managing MSK pain conditions, yet only 20\% of patients with LBP were given advice and education in a primary care setting. ${ }^{36}$

in their quality such as when developers have undisclosed interests $^{8}$ and a lack of transparency in their development. ${ }^{9}$ These problems are reflected in contemporary MSK pain CPGs. We recently reported that most MSK pain CPGs are of poor quality, use inconsistent terminology, over-represent some conditions (eg, low back pain [LBP] and osteoarthritis [ $\mathrm{OA}]$ ) and under-representation other conditions (eg, cervical and thoracic spine pain) and fail to outline how to implement CPGs. ${ }^{10}$ Nevertheless, we also identified a small number of higher quality CPGs that could be used to inform healthcare for MSK pain conditions.

There is increasing recognition that MSK pain conditions in different body areas share similarities with respect to mechanisms, prognostic factors and clinical course. ${ }^{11}{ }^{12}$ We were interested in whether there was common ground among CPGs for MSK pain. We were also interested in recommendations of care across MSK pain conditions that could potentially be applied across different levels of healthcare (ie, primary, tertiary and emergency). Therefore, we aimed to identify a common set of recommendations, obtained from contemporary high-quality CPGs, to assess and manage a broad range of MSK pain conditions.

\section{METHODS}

We undertook a systematic review and synthesis of contemporary MSK pain CPGs for three of the most common MSK pain sites ${ }^{13}$ : spinal pain (lumbar, thoracic and cervical spine), hip/knee pain including hip/knee OA and shoulder pain. The methods, including search strategy and selection processes, have been previously published. ${ }^{10}$ We selected CPGs published within 5 years of the original search date (since 2011). This cut-off date was selected as CPGs developed or updated within the previous 5 years better reflect up-to-date research evidence. ${ }^{4}$ The International Prospective Register of Systematic Reviews (PROSERO) registration number was CRD42016051653.

Our initial search included 34 CPGs that were developed between January 2011 and September 2016, for adults, in English, that reported development processes and were an original body of work. We excluded CPGs for: traumatic MSK pain, a single treatment modality (eg, surgery), traditional healing/ medicine, specific disease processes (eg, inflammatory arthropathies) or those that were private for-profit and required payment to access. Article titles/abstracts were initially screened by one investigator (IL). Full text articles were then reviewed independently by two investigators (IL and LW). In August 2017, one investigator (IL) updated the search to identify any newly developed CPGs (online online supplementary file 1). The updated list was reviewed for completeness by all authors. Ten additional CPGs were included.

\section{Appraisal}

Three investigators (IL, LW and RW) independently appraised the quality of CPGs using the Appraisal of Guidelines for Research and Evaluation II (AGREE-II) instrument. The AGREE II instrument is the most widely used generic instrument to measure the development and reporting of CPGs and has acceptably high construct validity (significant differences in 18 of 21 item scores between high/low quality CPGs) ${ }^{14}$ and reliability (item internal consistency between 0.64 and 0.89). ${ }^{14} 15$

\section{Analysis}

Using AGREE PLUS on the AGREE II website ${ }^{16}$ individual item scores, domain scores (scope and purpose, stakeholder involvement, rigour of development, clarity of presentation, applicability and editorial independence) and overall AGREE II scores were calculated for each CPG. AGREE PLUS calculates domain and overall scores as a percentage of the maximum possible score. Data were entered and analysed using SPSS (IBM SPSS Statistics V.24.0). Means and SD for each of the 23 items (1-7 scale) and six domain scores (percentage) were calculated. Inter-rater agreement was determined using intraclass correlation coefficients (ICCs) with a two-way random effects model for each domain and overall rating scores. We classified level of agreement as poor (ICC <0.40), fair (ICC $0.40-0.59$ ), good (ICC $0.60-0.74$ ) or excellent (ICC $0.75-1.00) .{ }^{17}$

Similarly to our previous study, ${ }^{10}$ we classified high-quality CPGs as those that had an AGREE II score equal to or greater than $50 \%$ of the maximum possible score in three domains: rigour of development (domain 3), editorial independence (domain 6) and stakeholder Involvement (domain 2). Our cutoff value of $50 \%$ is consistent with other studies, for example, ref ${ }^{18}$, and the AGREE II developer's recommendation that users decide on criteria for high and low quality CPGs based on the context of their work. ${ }^{16}$

\section{CPG synthesis and identifying consistent recommendations}

Synthesis consisted of four stages: extracting CPG recommendations, classifying recommendations, developing a narrative summary and, where possible, identifying consistent/common recommendations across MSK conditions. We also identified common recommendations within each MSK pain condition.

\section{Extracting recommendations}

Recommendations from high-quality CPGs were extracted independently by two reviewers (IL and either RW or LW). 
Table 1 Recommendation classification, definition and examples of terminology for each classification

\begin{tabular}{|c|c|c|}
\hline $\begin{array}{l}\text { Recommendation } \\
\text { classification }\end{array}$ & Definition & Examples of terminology \\
\hline 'Should do' & $\begin{array}{l}\text { 'Should do' recommendations were those that should be applied in all circumstances } \\
\text { unless there is a rationale not to. These were based on strong evidence, for example, } \\
\text { multiple high-quality studies reporting clinically relevant positive effects, benefits that } \\
\text { outweigh risks or when in the opinion of CPG development group members that the } \\
\text { benefits were unequivocal. }\end{array}$ & $\begin{array}{l}\text { 'Strong recommendation', for example, } \\
\text { 'should' occur. }{ }^{2326}{ }^{31}{ }^{12527} \text { and }\end{array}$ \\
\hline 'Could do' & $\begin{array}{l}\text { 'Could do' recommendations could be applied depending on the circumstances of } \\
\text { individual patients. They were usually based on consistent evidence from multiple lesser } \\
\text { quality studies or one high quality study and where benefits outweigh harms. }\end{array}$ & $\begin{array}{l}\text { 'Considered', for example, }{ }^{232529} \text { 'may include', }{ }^{26} \text { 'recommend', } \\
\text { 'practitioner might' and 'suggest'. }{ }^{21}\end{array}$ \\
\hline 'Do not do' & $\begin{array}{l}\text { 'Do not do' recommendations applied when there was strong evidence of no benefit } \\
\text { and/or harms outweighing benefits. Two CPGs provided strong and weak 'do not do' } \\
\text { recommendations }{ }^{2930} \text { that were combined for the purposes of our review. }\end{array}$ & $\begin{array}{l}\text { 'Do not offer', for example, }{ }^{27} 29 \text { 'should refrain from', }{ }^{28} \text { ' do not } \\
\text { routinely offer', }{ }^{29} \text { 'not appropriate }{ }^{22} \text { and 'should not'. }{ }^{28}\end{array}$ \\
\hline 'Uncertain' & $\begin{array}{l}\text { 'Uncertain' applied when there was no recommendation for or against a practice, } \\
\text { because of incomplete or inconsistent research findings. Not all CPGs provided uncertain } \\
\text { recommendations. }\end{array}$ & $\begin{array}{l}\text { 'Inconclusive' or 'we are unable to recommend for or } \\
\text { against', }{ }^{21} \text { 'inconclusive evidence }{ }^{127} \text { or 'uncertain'. }{ }^{22}\end{array}$ \\
\hline
\end{tabular}

CPGs, clinical practice guidelines.

The evidence supporting each recommendation, as reported by each CPG, was recorded (eg, level of evidence and strength of recommendation). Extracted recommendations were compared and discrepancies resolved through discussion and consensus.

\section{Classifying recommendations}

Two reviewers independently classified the recommendations. In order to accommodate the varying terminology used in different CPGs, we classified recommendations as 'should do', 'could do', 'do not do' or 'uncertain' (table 1). CPGs for which there were no recommendations in a particular topic, that is, it was out-of-scope of the CPG and was not included, were not classified.

\section{Narrative summary}

A narrative summary was developed initially by one author (IL) and then reviewed and refined by all authors. This included grouping recommendations into theme areas. This was a deviation from our original PROSPERO protocol. In our original protocol, we proposed that two authors would independently undertake initial thematic analysis. The author group included three academic and practising physiotherapists (IL, RW and PPBO), two MSK pain researchers (CGM and LS), an indicator development researcher (LW), a specialist emergency care physician (YN), a senior medical officer in emergency medicine (MG) and a pain medicine physician (RG). We undertook multiple rounds of review with the summary refined following each round. For example, recommendations were reorganised with manual therapy and surgery initially classified under 'physical therapies' and 'interventions', and following review, classified under 'passive interventions - non-invasive' and 'passive interventions - invasive'.

\section{Identifying consistent recommendations}

We identified consistent recommendations when there was a majority of 'should do' or 'do not do' recommendations and no conflicting recommendations in CPGs. Consistent recommendations applied across at least three MSK pain conditions. Additionally, we identified common recommendations within single MSK pain conditions based on the same criteria, a majority of 'should do' or 'do not do' recommendations in CPGs and no conflicting recommendations. We did not identify common recommendations when recommendations were weaker, that is, 'could do', 'uncertain' or there were conflicting recommendations.

\section{RESULTS}

Our searches, including an updated search, identified 6232 discrete records, from which 44 CPGs (34 CPGs initial search and 10 CPGs updated search) were selected for inclusion (figure 1). Fifteen of the included CPGs were for LBP, 14 were for OA, 6 for shoulder conditions and 5 for the neck. We included single CPGs for the neck/thoracic spine, knee, 'musculoskeletal injuries' and lower limb (online supplementary file 2).

\section{Characteristics of included CPGs}

With the exception of CPGs from Malaysia ${ }^{19}$ and the Philippines, ${ }^{20}$ all CPGs originated or involved panel members from high-income countries. CPGs were from 11 individual countries and 'international' collaborations, involving authors from multiple countries. Most were from the USA $(n=17)$, 'international' $(n=10)$ and Canada $(n=6)$. The Netherlands, UK and Italy each contributed two CPGs (online supplementary file 2).

CPGs from the USA were most commonly for LBP $(n=8)$, 'international' CPGs were most commonly for OA $(n=7)$, and half of Canadian CPGs were for neck pain $(n=3)$. The majority of CPGs were developed by medical societies $(n=23,52.3 \%)$ which were either related to a profession/specialty group, ${ }^{21}$, or MSK condition of interest, ${ }^{\mathrm{f}}$ or example, ref ${ }^{22}$.

\section{Appraisal of CPGs: inter-rater agreement}

Inter-rater agreement was 'fair' for scope and purpose (domain 1), 'good' for clarity of presentation (domain 4) and 'excellent' for all other domains and overall AGREE II score (table 2).

\section{Appraisal of CPGs: quality}

The mean (SD) AGREE II scores for each item, domain and overall scores across all guidelines are displayed in online supplementary file 3 . The domain with the lowest mean score was 'Applicability' $(27.6 \%, \mathrm{SD}=18.3)$, and the highest mean score was for 'Scope and Purpose' (73.7\%, SD=13.8).

The AGREE II scores for each CPG are displayed in online supplementary file 4 . In our previous review, ${ }^{10}$ eight high-quality MSK pain CPGs were identified for OA, ${ }^{21-24} \mathrm{LBP}^{25}{ }^{26}$ neck pain, ${ }^{27}$ and shoulder pain. ${ }^{28}$ Of the 10 additional CPGs identified in our updated search, three were rated as high-quality and 


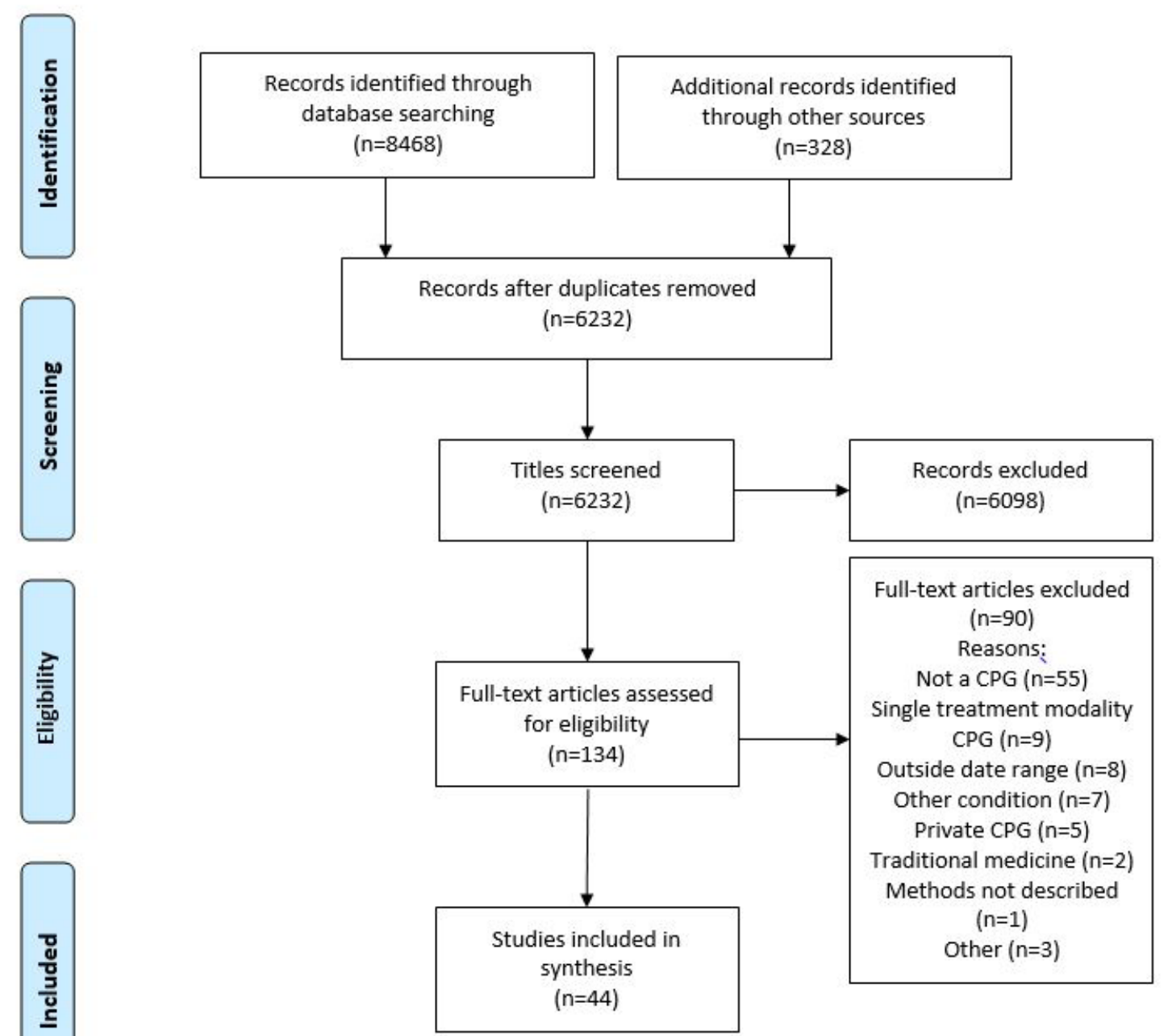

Figure 1 PRISMA flow diagram. CPG, clinical practice guidelines; PRISMA, Preferred Reporting Items for Systematic Reviews and Meta-Analyses.

included in our synthesis, including two for $\mathrm{LBP}^{2930}$ and one for neck pain $^{31}$ (online supplementary file 3 ).

\section{Consistent recommendations}

Following synthesis (table 3, online supplementary file 5), 11 common/consistent recommendations were identified across MSK pain conditions (box 2).

\begin{tabular}{ll}
\hline $\begin{array}{l}\text { Table } 2 \\
\text { scores }\end{array}$ & Inter-rater agreement for AGREE II domains and overall \\
\hline Domain & ICC $(95 \% \mathrm{Cl})$ \\
\hline Scope and purpose & $0.480(0.08$ to 0.71$)$ \\
\hline Stakeholder involvement & $0.797(0.62$ to. 09$)$ \\
\hline Rigour of development & $0.915(0.85$ to. 095$)$ \\
\hline Clarity of presentation & $0.734(0.56$ to 0.85$)$ \\
\hline Applicability & $0.792(0.64$ to 0.88$)$ \\
\hline Editorial independence & $0.886(0.81$ to 0.93$)$ \\
\hline Overall rating & $0.827(0.72$ to 0.90$)$ \\
\hline $\begin{array}{l}\text { AGREE, Appraisal of Guidelines for Research and Evaluation; ICC, intraclass } \\
\text { correlation coefficients. }\end{array}$
\end{tabular}

Care should be patient centred. This includes care that responds to the individual context of the patient, employs effective communication and uses shared decision-making processes

All CPGs described delivery of patient-centred care, most commonly as an introductory principle and sometimes specifically articulated within recommendations. Patient-centred care included providing individualised care based on the context of the patient and their preferences, ${ }^{21-26} 28-31$ shared decision making, ${ }^{2426}$ using effective communication ${ }^{21242628}$ and/or an explicit discussion of 'patient centred care'. ${ }^{27} 29$

Practitioners should screen patients to identify those with a high likelihood of serious pathology/red flag conditions

Seven CPGs offered 'should do' recommendations to screen patients for possible serious or structural pathology/'red flag' conditions during an initial assessment. ${ }^{24-2931}$ Examples included suspicion of infection, malignancy, fracture, inflammatory causes of pain, severe and progressive neurological deficit (including cauda equina syndrome) and serious conditions that masquerade as MSK pain, for example, aortic aneurysm. The National Institute for Health and Care Excellence (NICE) OA CPG identified 


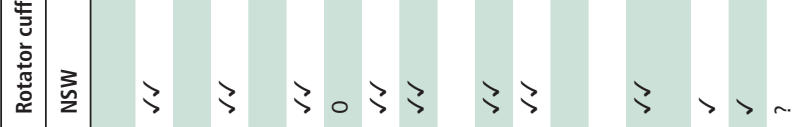

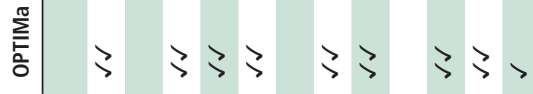

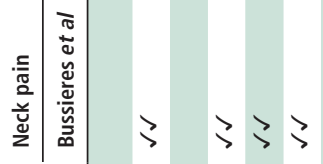

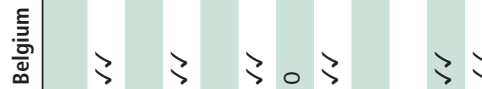

$33>$

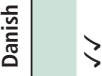

\o

>

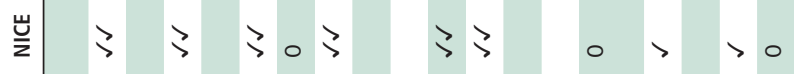

$00>>00>3$

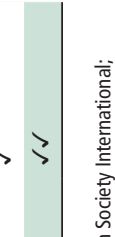

$\underset{\sim}{\square}$

$\frac{\infty}{0}$

을

잉

$\stackrel{\vec{F}}{\stackrel{9}{+}}$

$>$

$\frac{\overline{0}}{\overline{\frac{C}{0}}}$

$\frac{\widehat{\nabla}}{\stackrel{2}{2}}$

$\stackrel{\infty}{\circ}$

$\overrightarrow{\vec{\omega}}$

0
$\frac{0}{6}$
$\frac{0}{0}$
$\frac{1}{0}$
0
$\hat{~}$
0
0
0
$\dot{0}$
0
0
$\infty$
$\infty$
0
0

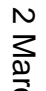

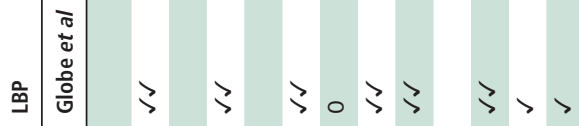

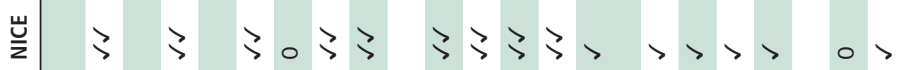

\ฺ

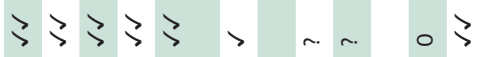

誉 \

ฮ管 引

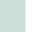


Box 2 Consistent recommendations across musculoskeletal (MSK) pain conditions

1. Care should be patient centred. This includes care that responds to the individual context of the patient, employs effective communication and uses shared decision-making processes.

2. Screen patients to identify those with a higher likelihood of serious pathology/red flag conditions.

3. Assess psychosocial factors.

4. Radiological imaging is discouraged unless:

i. Serious pathology is suspected.

ii. There has been an unsatisfactory response to conservative care or unexplained progression of signs and symptoms.

iii. It is likely to change management.

5. Undertake a physical examination, which could include neurological screening tests, assessment of mobility and/or muscle strength.

6. Patient progress should be evaluated including the use of outcome measures.

7. Provide patients with education/information about their condition and management options.

8. Provide management addressing physical activity and/or exercise.

9. Apply manual therapy only as an adjunct to other evidencebased treatments.

10. Unless specifically indicated (e.g. red flag condition), offer evidence-informed non-surgical care prior to surgery.

11. Facilitate continuation or resumption of work.

important differential diagnoses as gout, other inflammatory arthritides, septic arthritis and malignancy (bone pain). ${ }^{24}$

\section{Psychosocial factors should be assessed}

Nine CPGs, including four for LBP, 25262930 two for neck pain, ${ }^{2731}$ two for $\mathrm{OA}^{23} 24$ and one for rotator cuff disorders ${ }^{28}$ recommended assessing psychosocial factors. These factors included 'yellow flags', mood/emotions (depression and anxiety), fear/kinesiophobia and recovery expectations. Two LBP CPGs ${ }^{25} 29$ recommended using the STarT Back ${ }^{32}$ or Orebro Musculoskeletal Screening tools ${ }^{33}$ to identify psychosocial prognostic risk factors and subsequently match care to the level of patient risk.

\section{Radiological imaging is discouraged unless: (1) serious pathology} is suspected, (2) there has been an unsatisfactory response to conservative care or unexplained progression of signs and symptoms or (3) imaging is likely to change management

Six CPGs discouraged routine use of radiological imaging. ${ }^{24-2628-30}$ In the case of LBP, routine imaging was discouraged unless serious pathology is suspected (ie, 'red flag' conditions), findings are likely to 'change management' (such as if administration of an epidural or spinal surgery is under consideration ${ }^{25}{ }^{29}$ ), or if there has been a limited response to conservative care. ${ }^{26}$ Two LBP CPGs recommended explaining to patients that imaging may not be needed. ${ }^{25} 29$ In contrast one chiropractic CPG recommended consideration of imaging if there is 'suspicion of an underlying anatomical anomaly, such as spondylolisthesis, moderate to severe spondylosis' or 'mechanical instability'. ${ }^{26}$ The NICE CPG encouraged clinical diagnosis of OA without imaging. ${ }^{24}$
Assessment should include physical examination. Physical examination could include neurological screening tests, mobility and/or muscle strength

Seven CPGs recommended undertaking a physical examination when assessing MSK pain conditions. ${ }^{23-29}$ Physical assessments included mobility/movement, strength, ${ }^{23}{ }^{28}$ position and proprioception. ${ }^{23} \mathrm{CPGs}$ for spinal pain also recommended tests of neurological function. ${ }^{26} 2729$ The purpose of physical examination tests were to assist in the diagnosis or classification of MSK pain disorders. For the shoulder, this included differentiating between tendon, articular or referred pain origin. ${ }^{28}$ Neurological testing in the lumbar spine was used to differentiate pain of radicular origin. ${ }^{29}$ Physical examination procedures in the cervical spine were recommended to classify neck pain disorders into grades I-III. ${ }^{27}$ One CPG noted a lack of empirical support for physical assessments, although concluded that 'the repercussions of not performing an examination would lead to dissatisfaction and unwarranted demand for tests or further referrals'. ${ }^{25}$

Patient progress should be evaluated, including the use of validated outcome measures

Five CPGs recommended evaluating patient progress ${ }^{23} 2426-28$ and four of these recommended clinicians use validated outcome measures. Recommended outcome measures included a sevenpoint patient self-rated recovery question, ${ }^{27}$ pain intensity, ${ }^{26}$ functional capacity/activities of daily living ${ }^{2628}$ and/or quality of life. $^{24}$

All patients should be provided with education/information about their condition and management options

Ten CPGs recommended providing education or information either to: (1) encourage self-management ${ }^{22} 23252729-31$ and/or (2) inform/reassure patients about the condition or management (eg, prognosis and psychosocial aspects). ${ }^{23}{ }^{24}{ }^{26-31}$ In all CPGs, education/information was recommended as part of a package of care alongside other treatments, and a number of CPGs emphasised the need for education to be individualised based on patient need. 23252930

Patients should receive management that addresses physical activity and/or exercise

All CPGs included recommendations relating to activity/exercise, with the strength of recommendation varying according to the type of activity/exercise and between different MSK conditions. CPGs for OA, ${ }^{21-24}$ LBP $^{25262930}$ and neck pain ${ }^{2731}$ made general recommendations for physical activity/exercise including maintenance of activity/'normal' physical activity, aerobic exercises, 'exercise' and/or 'general exercise'. CPGs for OA had strong recommendations for specific exercise, most commonly strengthening, ${ }^{21-24}$ as well as mobility (eg, range of motion and stretching), ${ }^{22}{ }^{23}$ water-based exercises, ${ }^{22}{ }^{23}$ neuromuscular education $^{21}$ or tai chi. ${ }^{22}$ For rotator cuff disorders, one CPG recommended initial treatment with prescribed exercise, such as stretching, flexibility and strengthening. ${ }^{28}$ Three CPGs for LBP recommended supervised exercise. ${ }^{25} 2630$ neckpain and associated disorders (NAD) CPGs recommended supervised graded neck strengthening for NAD grade III and as part of multimodal care for NAD grades I-II. ${ }^{27} 31$

If used, manual therapy should be applied only in conjunction with other treatments

Manual therapy was a 'could do' recommendation for seven $\mathrm{CPGs}^{24} 25$ 27-31 and a 'should do' recommendation for one. ${ }^{26}$ 
However when included, manual therapy was consistently recommended as a component of multimodal care, in conjunction with other management strategies, including exercise, psychological therapy, information/education, and activity advice rather than a stand-alone treatment.

Unless specifically contraindicated (eg, 'red flag' condition[s]), offer evidence-informed non-surgical care prior to surgery

Four CPGs recommended that patients be provided non-surgical treatments prior to consideration of surgery. ${ }^{24252829}$ The CPG for rotator cuff disorders was the only to specify a trial duration for non-surgical care, recommending surgical review if 3 months of non-surgical management had been unsuccessful and/or following non-surgical care there was a symptomatic full thickness rotator cuff tear on review. ${ }^{28}$

\section{Facilitate continuation or resumption of work}

Five CPGs offered a 'should do' recommendation for re-engagement with or continuation of work for patients with MSK pain, including neck pain, ${ }^{27} \mathrm{OA},{ }^{23}$ rotator cuff syndrome ${ }^{28}$ and LBP. $^{29}$ One CPG for LBP offered a 'could do' recommendation to advise patients to stay active, avoid inactivity and gradually increase activity levels, including work involvement. ${ }^{30}$ Three CPGs emphasised early return to work ${ }^{29}$ including engagement with vocational rehabilitations services, communication between worker, employer and health provider and planning processes to facilitate return to work. ${ }^{28}$

\section{Single MSK conditions and conflicting recommendations}

We also identified common recommendations for single MSK pain conditions including seven for LBP, four for OA and one for NAD (box 3, online supplementary file 5).

Conflicting recommendations for CPGs both between MSK pain conditions and within CPGs for a specific condition were

Box 3 Consistent recommendations within single musculoskeletal (MSK) pain conditions

Osteoarthritis (OA)

- Offer self-management programmes.

- Provide interventions targeting weight loss to people with $\mathrm{OA}$ who are overweight or obese.

- Do not use glucosamine or chondroitin for disease modification.

- Do not undertake knee arthroscopic lavage and debridement unless there is a rationale (such as mechanical knee locking).

\section{Low back pain}

- Do not offer paracetamol as a single medication.

- Do not offer opioids for chronic LBP.

- Do not offer selective serotonin reuptake inhibitors, serotonin-norepinephrine reuptake inhibitors, tricyclic antidepressants or anticonvulsants for LBP.

- Do not offer rocker shoes or foot orthotics.

- Do not offer disc replacement.

- Only offer spinal fusion if part of a randomised controlled trial.

- Spinal injections (eg, facet joint injections, medical branch blocks, intradiscal injections, prolotherapy and trigger point injections) should not be used for LBP.

Neck pain

- Neck pain disorders should be classified as grades I-IV. noted for medication prescription (including paracetamol and non-steroidal anti-inflammatory drugs [NSAIDs]), electrotherapy, the use of braces and orthoses and acupuncture. There were also conflicting recommendations regarding the prescription of opioids including 'could do', 'do not do' and 'uncertain' recommendations. CPGs that offered 'could do' recommendations advocated caution, including careful consideration of risk and benefits, ${ }^{24}{ }^{30}$ use of opioids 'for the shortest period possible ${ }^{29}$ and 'not routinely'. ${ }^{25}$

\section{DISCUSSION}

We identified 11 common recommendations for MSK pain care (box 2) that applied to patients with OA, LBP, neck pain and shoulder pain. These recommendations may provide clinicians, healthcare managers, funders, policy makers and researchers with a simple and clear consensus of current MSK pain priorities. In turn, this could help address the variable quality of clinical care delivered for MSK pain conditions. ${ }^{34-36}$

The common recommendations could be used in a number of ways to improve care. First, they could guide consumers in making informed healthcare decisions or assist them to identify when they are receiving suboptimal care. Second, clinicians could apply the recommendations to guide care decision making, to identify areas for learning and development and to assess their practice. Third, health services could assess the quality of care by applying them as minimum standards during clinical audit. Finally, a broader strategy (eg, for researchers, health planners/ policy makers or funders) could be the continued development of the common recommendations into a set of quality indicators that could be used for reporting or to benchmark care quality. The recommendations could be used as a preliminary set of standards that could be refined via a rigorous, structured process. ${ }^{37}$

Our findings are similar to a recent review by Babatunde et al that was a synthesis of MSK pain management in primary care. ${ }^{11}$ Recommended care included self-management advice, education and exercise as first-line treatments for MSK pain. The authors recommended short-term NSAIDs and opioids for pain relief provided risks and harms were considered. There was limited evidence for aids and devices (eg, taping and braces) and passive treatments (eg, acupuncture, transcutaneous electrical nerve stimulation and laser) and inconsistent evidence for surgery (equivalent to conservative care options in the longer term). ${ }^{11}$ In contrast to our review, the authors reported support for psychosocial interventions, especially for those at risk of poor prognosis. We found that while CPGs in our review universally recommended psychosocial assessment, only LBP and neck pain CPGs reported on psychological treatments ('could do' recommendations for psychological therapies for LBP and contradictory recommendations for neck pain). Although our review included more recent CPGs, for example, ${ }^{25-27}{ }^{29-31} \mathrm{CPGs}$ for other common MSK pain conditions including OA and hip pain, Babatunde $e \mathrm{al}^{11}$ included other sources of literature such as trials and systematic reviews. Psychological-informed management for MSK pain is an area that is currently lacking in CPGs.

Our review identified CPGs for LBP, OA, NAD and rotator cuff disorders. Arguably the common recommendations are relevant to other MSK pain conditions. For example, in tendinopathy, psychosocial factors are known predictors of outcome ${ }^{38}$; radiological imaging is of questionable clinical utility because of a moderate relationship between symptoms and outcome, ${ }^{39}$ and high value nonsurgical approaches to management such as education and exercise form the cornerstones of care. ${ }^{40} 41$ Although high-quality CPGs are currently lacking for other MSK pain conditions, we speculate 
that the consistent recommendations could be applied broadly to managing non-traumatic MSK pain.

Due to the potential for harm, an area we were interested in was opioid prescribing. We were unable to identify a consensus among CPGs relating to opioids, and there were conflicting recommendations both within and across MSK pain conditions. While there were conflicting views, almost all CPGs urged caution and/or discouraged opioid use. Recent research has reported no additional benefit of an opioid over a NSAID for acute $\mathrm{LBP}^{42}$ and further efficacy studies are underway. ${ }^{43}$ Recent opioid-prescribing CPGs for patients with chronic pain ${ }^{45}$ recommend risk assessment to identify those at higher likelihood of opioid-related harms. Although outside the scope of this review, these recommendations should be considered by clinicians.

Our findings were similar to recent reviews of LBP management in emergency care. ${ }^{46}{ }^{47}$ In emergency care, clinicians should use 'red flags' to assess the likelihood of serious pathology, apply judicious use of radiological imaging, identify psychosocial risk factors, provide targeted education and reassurance, offer exercise, cold and heat and promote early return to work and function. ${ }^{46}$ The similarities of these recommendations and our findings suggest they could be applied in varied healthcare settings.

Results of our updated search reinforced our previous findings $^{10}$ that most MSK pain CPGs were of poor quality, which is a waste of resources. In addition to directing resources to fewer, higher quality CPGs, another way to reduce waste is for developers to focus on gaps in recommendations, areas for which there is currently limited consensus, and on other MSK pain conditions. As previously described, medication prescribing and psychologically oriented management of MSK pain are areas that lack research or have not been addressed by CPGs to date. CPGs targeting thoracic pain and non-OA knee pain are MSK pain areas that are lacking.

\section{Limitations}

The AGREE II instrument reflects methodological processes, not necessarily content, and scores may reflect reporting rather than methodological quality. The AGREE II developer's suggest research groups identify their own criteria for CPG quality. ${ }^{16}$ Our group defined high-quality CPGs as those who scored higher than 50\% in three AGREE II domains. Although consistent with the AGREE II developer's recommendations, investigator-developed criteria have the potential for bias by including or excluding CPGs based on non-empirically derived criteria. While a cut-off value of $50 \%$ is consistent with other studies, for example, ${ }^{18}$ our criteria is somewhat generous when compared with some other reviewers for example. ${ }^{48}$ CPGs were appraised by three authors and ideally four should be used. ${ }^{14}$ Our research group was interprofessional; CPG appraisers were academic and clinical physiotherapists (IL and RW) and an indicator development researcher also with a background in physiotherapy (LW). Our synopsis of CPGs and interpretation of their recommendations (eg, as 'should do', 'could do' and so on), due to variations in the reporting and language of CPGs, relied on the interpretation of the research group. This is an inherent issue where there is heterogeneity in the way CPGs are conceptualised and their recommendations and underpinning evidence are presented. For example, some CPGs offer broad generalised recommendations ${ }^{26}$ compared with others that address specific, highly targeted questions. ${ }^{31}$ To account for this, we have ensured our interpretation and methodological steps have been clearly reported. As always, the search strategy may have failed to identify all relevant documents, and we only reviewed CPGs available in English. Nevertheless, our involvement of a reference librarian during searching and the experience of the research group means we are confident that relevant CPGs were included.

\section{CONCLUSION}

Variation in the quality of care is a barrier to providing high value MSK pain care. CPGs are an important tool to address MSK pain care quality. We identified 11 consistent recommendations (box 2) for the management of MSK pain conditions. These recommendations can be used by consumers, clinicians and at health services and policy levels to improve the quality of MSK pain care. Optimising the implementation of these recommendations comprises the next challenge.

\section{Author affiliations}

${ }^{1}$ WA Centre for Rural Health, University of Western Australia, Geraldton, Western Australia, Australia

${ }^{2}$ Centre for Population Health Research, University of South Australia Division of Health Sciences, Adelaide, South Australia, Australia

${ }^{3}$ School of Physiotherapy and Exercise Science, Curtin University, Perth, Western Australia, Australia

${ }^{4}$ Pain Management Centre, Sir Charles Gardner Hospital, Perth, Western Australia, Australia

${ }^{5}$ Centre for Clinical Research in Emergency Medicine, Harry Perkins Institute of Medical Research, Perth, Western Australia, Australia

${ }^{6}$ Emergency Department, Fiona Stanley Hospital, Murdoch, Western Australia, Australia

${ }^{7}$ Emergency Department, Geraldton Hospital, Geraldton, Western Australia, Australia ${ }^{8}$ School of Physiotherapy and Curtin Health Innovation Research Institute, Curtin University, Bentley, Western Australia, Australia

${ }^{9}$ School of Public Health, Sydney Medical School, The University of Sydney, Sydney, New South Wales, Australia

${ }^{10}$ Department of Physiotherapy, Curtin University, Perth, Western Australia, Australia

Acknowledgements We acknowledge Anne Smith for statistical assistance.

Contributors All authors were involved in the conception, design and interpretation of data. IL, LW and RW performed the data analysis and initial interpretation. IL was responsible for initial writing and drafting of the article, which was reviewed by all authors. All authors revised critically for important intellectual content and approved the final version to be submitted.

Funding IL is funded by an Australian National Health and Medical Research Council Early Career Fellowship (APP1090403). CGM's fellowship (APP1103022) is funded by Australia's National Health and Medical Research Council, and his research is supported by a Program Grant (APP1113532) and CRE Grant (APP1134856). LW works on a project funded by a National Health and Medical Research Council Program Grant (APP1054146).

Competing interests None declared.

Patient consent for publication Not required.

Provenance and peer review Not commissioned; externally peer reviewed.

\section{ORCID iDs}

Ivan Lin http://orcid.org/0000-0001-6901-2569

Leon Straker http://orcid.org/0000-0002-7786-4128

\section{REFERENCES}

1 Vos T, Abajobir AA, Abate KH, et al. Global, regional, and national incidence, prevalence, and years lived with disability for 328 diseases and injuries for 195 countries, 1990-2016: a systematic analysis for the Global Burden of Disease Study 2016. The Lancet 2017;390:1211-59.

2 Buchbinder $\mathrm{R}$, van Tulder $\mathrm{M}$, Öberg B, et al. Low back pain: a call for action. Lancet 2018;391:2384-8.

3 Eccles MP, Grimshaw JM, Shekelle $\mathrm{P}$, et al. Developing clinical practice guidelines: target audiences, identifying topics for guidelines, guideline group composition and functioning and conflicts of interest. Implement Sci 2012;7:60.

4 IOM (Institute of Medicine). Clinical practice guidelines we can trust. Washington (DC): National Academies Press, 2011.

5 Childs JD, Fritz JM, Wu SS, et al. Implications of early and guideline adherent physical therapy for low back pain on utilization and costs. BMC Health Serv Res 2015;15:15.

6 Rutten GM, Degen S, Hendriks EJ, et al. Adherence to clinical practice guidelines for low back pain in physical therapy: do patients benefit? Phys Ther 2010;90:1111-22. 
7 Baiardini I, Braido F, Bonini M, et al. Why do doctors and patients not follow guidelines? Curr Opin Allergy Clin Immunol 2009;9:228-33.

8 Williams MJ, Kevat DA, Loff B, et al. Conflict of interest guidelines for clinical guidelines. Med J Aust 2011;195:442-5.

9 Scott IA, Guyatt GH. Clinical practice guidelines: the need for greater transparency in formulating recommendations. Med J Aust 2011;195:29.

10 Lin I, Wiles LK, Waller R, et al. Poor overall quality of clinical practice guidelines for musculoskeletal pain: a systematic review. Br J Sports Med 2018:52:337-43.

11 Babatunde 00, Jordan JL, Van der Windt DA, et al. Effective treatment options for musculoskeletal pain in primary care: a systematic overview of current evidence. PLoS One 2017:12:e0178621.

12 Henschke N, Ostelo RW, Terwee CB, et al. Identifying generic predictors of outcome in patients presenting to primary care with nonspinal musculoskeletal pain. Arthritis Care Res 2012;64:1217-24.

13 Smith E, Hoy DG, Cross M, et al. The global burden of other musculoskeletal disorders: estimates from the Global Burden of Disease 2010 study. Ann Rheum Dis 2014;73:1462-9.

14 Brouwers MC, Kho ME, Browman GP, et al. Development of the AGREE II, part 2: assessment of validity of items and tools to support application. CMAJ 2010;182:E472-8.

15 Brouwers MC, Kho ME, Browman GP, et al. Development of the AGREE II, part 1: performance, usefulness and areas for improvement. Can Med Assoc J 2010;182:1045-52.

16 AGREE Enterprise. AGREE: advancing the science of practice guidelines: Canadian Institutes of Health Research. 2014 http://www.agreetrust.org/ (Accessed 29 Mar 2016).

17 Cicchetti DV. Guidelines, criteria, and rules of thumb for evaluating normed and standardized assessment instruments in psychology. Psychol Assess 1994;6:284-90.

18 Bouwmeester W, van Enst A, van Tulder M, et al. Quality of low back pain guidelines improved. Spine 2009:34:2562-7.

19 Rosman A, Ismail A, Zain M, et al. Management of osteoarthritis. 2nd edn. Putrajaya, Malaysia: Ministry of Health Malaysia, 2013.

20 Philippine Academy of Rehabilitation Medicine. Low back pain management guideline. Quezon City, Philippines: Philippine Academy of Rehabilitation Medicine (PARM), 2012.

21 Jevsevar DS, Brown GA, Jones DL, et al. Treatment of osteoarthritis of the knee: evidence-based guideline - second edition. Rosemont, IL: American Academy of Orthopaedic Surgeons, 2013.

22 McAlindon TE, Bannuru RR, Sullivan MC, et al. OARSI guidelines for the non-surgical management of knee osteoarthritis. Osteoarthritis Cartilage 2014:22:363-88

23 Fernandes L, Hagen KB, Bijlsma JW, et al. EULAR recommendations for the nonpharmacological core management of hip and knee osteoarthritis. Ann Rheum Dis 2013;72:1125-35.

24 National Institute for Health and Clinical Excellence (NICE). Osteoarthritis: care and management (CG 177). London: National Institute for Health and Clinical Excellence, 2014.

25 National Institute for Health and Clinical Excellence (NICE). Low back pain and sciatica in over 16s: assessment and management (NG59). London: National Institute for Health and Clinical Excellence, 2016

26 Globe G, Farabaugh RJ, Hawk C, et al. Clinical practice guideline: chiropractic care for low back pain. J Manipulative Physiol Ther 2016;39:1-22.

27 Côté P, Wong JJ, Sutton D, et al. Management of neck pain and associated disorders: a clinical practice guideline from the Ontario Protocol for Traffic Injury Management (OPTIMa) Collaboration. Eur Spine J 2016;25:2000-22.

28 Hopman K, Krahe L, Lukersmith S, et al. Clinical practice guidelines for the management of rotator cuff syndrome in the workplace. Port Macquarie (Australia): University of New South Wales, 2013:80.

29 Van Wambeke P, Desomer A, Ailiet L, et al. Low back pain and radicular pain: assessment and management. KCE Report 2017:287.

30 Stochkendahl MJ, Kjaer P, Hartvigsen J, et al. National Clinical Guidelines for non-surgical treatment of patients with recent onset low back pain or lumbar radiculopathy. Eur Spine J 2018;27.

31 Bussières AE, Stewart G, Al-Zoubi F, et al. The treatment of neck pain-associated disorders and whiplash-associated disorders: a clinical practice guideline. J Manipulative Physiol Ther 2016;39:523-64.

32 Hill JC, Whitehurst DG, Lewis M, et al. Comparison of stratified primary care management for low back pain with current best practice (STarT Back): a randomised controlled trial. Lancet 2011;378:1560-71.

33 Linton SJ, Nicholas M, MacDonald S. Development of a short form of the Örebro Musculoskeletal Pain Screening Questionnaire. Spine 2011;36:1891-5.

34 Runciman WB, Hunt TD, Hannaford NA, et al. CareTrack: assessing the appropriateness of health care delivery in Australia. Med J Aust 2012;197:100-5

35 Buchbinder R, Staples MP, Shanahan EM, et al. General practitioner management of shoulder pain in comparison with rheumatologist expectation of care and best evidence: an Australian national survey. PLoS One 2013;8:e61243.

36 Williams CM, Maher CG, Hancock MJ, et al. Low back pain and best practice care: a survey of general practice physicians. Arch Intern Med 2010;170:271-7.
37 Campbell SM, Braspenning J, Hutchinson A, et al. Research methods used in developing and applying quality indicators in primary care. Qual Saf Health Care 2002;11:358-64.

38 Mallows A, Debenham J, Walker T, et al. Association of psychological variables and outcome in tendinopathy: a systematic review. Br J Sports Med 2017;51:743-8.

39 Khan KM, Forster BB, Robinson J, et al. Are ultrasound and magnetic resonance imaging of value in assessment of Achilles tendon disorders? A two year prospective study. Br J Sports Med 2003;37:149-53.

40 Coombes BK, Bisset L, Vicenzino B. Management of lateral elbow tendinopathy: one size does not fit all. J Orthop Sports Phys Ther 2015:45:938-49.

41 Grimaldi A, Fearon A. Gluteal tendinopathy: integrating pathomechanics and clinical features in its management. J Orthop Sports Phys Ther 2015:45:910-22.

42 Friedman BW, Dym AA, Davitt M, et al. Naproxen with cyclobenzaprine, oxycodone/ acetaminophen, or placebo for treating acute low back pain: a randomized clinical trial. JAMA 2015;314:1572-80.

43 Lin CW, McLachlan AJ, Latimer J, et al. OPAL: a randomised, placebo-controlled trial of opioid analgesia for the reduction of pain severity in people with acute spinal pain. Trial protocol. BMJ Open 2016;6:e011278.

44 Dowell D, Haegerich TM, Chou R. CDC Guideline for prescribing opioids for chronic pain-United States, 2016. JAMA 2016;315:1624-45.

45 Department of Veterans Affairs. VA/DoD clinical practice guideline for opioid therapy for chronic pain: Version. 2017

46 Strudwick K, McPhee M, Bell A, et al. Review article: Best practice management of low back pain in the emergency department (part 1 of the musculoskeletal injuries rapid review series). Emerg Med Australas 2018;30:18-35.

47 Machado GC, Rogan E, Maher CG. Managing non-serious low back pain in the emergency department: time for a change? Emerg Med Australas 2018:30:279-82

48 Armstrong JJ, Rodrigues IB, Wasiuta T, et al. Quality assessment of osteoporosis clinical practice guidelines for physical activity and safe movement: an AGREE II appraisal. Arch Osteoporos 2016;11:1-10.

49 Ivanova JI, Birnbaum HG, Schiller M, et al. Real-world practice patterns, health-care utilization, and costs in patients with low back pain: the long road to guidelineconcordant care. Spine J 2011;11:622-32.

50 Girish G, Lobo LG, Jacobson JA, et al. Ultrasound of the shoulder: asymptomatic findings in men. AJR Am J Roentgenol 2011;197:W713-19.

51 Adelani MA, Harris AH, Bowe TR, et al. Arthroscopy for knee osteoarthritis has not decreased after a clinical trial. Clin Orthop Relat Res 2016;474:489.

52 Judge A, Murphy RJ, Maxwell R, et al. Temporal trends and geographical variation in the use of subacromial decompression and rotator cuff repair of the shoulder in England. Bone Joint J 2014;96-B:70.

53 Ketola S, Lehtinen JT, Arnala I. Arthroscopic decompression not recommended in the treatment of rotator cuff tendinopathy: a final review of a randomised controlled trial at a minimum follow-up of ten years. Bone Joint J 2017;99B:799-805.

54 Beard DJ, Rees JL, Cook JA, et al. Arthroscopic subacromial decompression for subacromial shoulder pain (CSAW): a multicentre, pragmatic, parallel group, placebocontrolled, three-group, randomised surgical trial. The Lancet 2017.

55 Abdel Shaheed C, Maher CG, Williams KA, et al. Efficacy, tolerability, and dosedependent effects of opioid analgesics for low back pain: a systematic review and meta-analysis. JAMA Intern Med 2016:176:958.

56 Webster BS, Verma SK, Gatchel RJ. Relationship between early opioid prescribing for acute occupational low back pain and disability duration, medical costs, subsequen surgery and late opioid use. Spine 2007;32:2127-32.

57 Sullivan MD, Howe CQ. Opioid therapy for chronic pain in the United States: promises and perils. Pain 2013;154:594-100.

58 American Academy of Orthopaedic Surgeons. Management of osteoarthritis of the hip: evidence-based clinical practice guideline. Rosemont, Illinois, 2017.

59 Hochberg MC, Altman RD, April KT, et al. American College of Rheumatology 2012 recommendations for the use of nonpharmacologic and pharmacologic therapies in osteoarthritis of the hand, hip, and knee. Arthritis Care Res 2012;64:465-74.

60 Bruyère 0 , Cooper C, Pelletier JP, et al. An algorithm recommendation for the management of knee osteoarthritis in Europe and internationally: a report from a task force of the European Society for Clinical and Economic Aspects of Osteoporosis and Osteoarthritis (ESCEO). Semin Arthritis Rheum 2014;44:253-63.

61 Bruyère $\mathrm{O}$, Cooper $\mathrm{C}$, Pelletier $\mathrm{JP}$, et al. A consensus statement on the European Society for Clinical and Economic Aspects of Osteoporosis and Osteoarthritis (ESCEO) algorithm for the management of knee osteoarthritis-from evidence-based medicine to the real-life setting. Semin Arthritis Rheum 2016;45:S3-11.

62 Peter WF, Jansen MJ, Hurkmans EJ, et al. Physiotherapy in hip and knee osteoarthritis: development of a practice guideline concerning initial assessment, treatment and evaluation. Acta Reumatol Port 2011:36:268-81.

63 Brosseau L, Wells GA, Tugwell P, et al. Ottawa Panel evidence-based clinical practice guidelines for the management of osteoarthritis in adults who are obese or overweight. Phys Ther 2011:91:843-61.

64 Brosseau L, Wells GA, Pugh AG, et al. Ottawa Panel evidence-based clinical practice guidelines for therapeutic exercise in the management of hip osteoarthritis. Clin Rehabil 2016;30:935-46. 
65 Goodman F, Kaiser L, Kelley C, et al. VA/DoD clinical practice guideline for the nonsurgical management of hip and knee osteoarthritis: Department of Veterans Affairs, Department of Defense, 2014:126.

66 Qaseem A, Wilt TJ, McLean RM, et al. Noninvasive treatments for acute, subacute, and chronic low back pain: a clinical practice guideline from the American College of Physicians. Ann Intern Med 2017; 166:514.

67 Chou R, Deyo R, Friedly J, et al. Noninvasive treatments for low back pain. Portland, OR: Agency for Healthcare Research and Quality, AHRQ, 2016.

68 Delitto A, George SZ, Van Dillen L, et al. Low Back Pain: clinical practice guidelines linked to the international classification of functioning, disability, and health from the orthopaedic section of the American Physical Therapy Association. J Orthop Sports Phys Ther 2012;42:A1-57.

69 Lee J, Gupta S, Price C, et al. Low back and radicular pain: a pathway for care developed by the British Pain Society. Br J Anaesth 2013;111:112-20.

70 Cheng L, Lau K, Lam W, et al. Evidence-based guideline on prevention and management of low back pain in working population in primary care. The Hong Kong Practitioner 2012;34.

71 State of Colorado Department of Labor and Employment: Division of Worker's Compensation. Low back pain: medical treatment guidelines. Denver, Colorado, 2014:112.

72 Goertz M, Thorson D, Bonsell J, et al. Adult acute and subacute low back pain: Institute for Clinical Systems Improvement Updated, 2012.

73 Kreiner DS, Hwang SW, Easa JE, et al. An evidence-based clinical guideline for the diagnosis and treatment of lumbar disc herniation with radiculopathy. Spine J 2014;14:180-91.

74 Kreiner DS, Shaffer WO, Baisden JL, et al. An evidence-based clinical guideline for the diagnosis and treatment of degenerative lumbar spinal stenosis (update). Spine $J$ 2013;13:734-43.

75 Matz PG, Meagher RJ, Lamer T, et al. Guideline summary review: an evidencebased clinical guideline for the diagnosis and treatment of degenerative lumbar spondylolisthesis. Spine J 2016;16:439-48.

76 Toward Optimized Practice (TOP) Program. Evidence-informed primary care management of low back pain: clinical practice guidelines. 3rd edn. Alberta, Canada: Institute of Health Economics, 2015:49.

77 State of Colorado Department of Labor and Employment: Division of Worker's Compensation. Shoulder injury: medical treatment guidelines. Denver, Colorado, 2015:164.
78 Diercks R, Bron C, Dorrestijn O, et al. Guideline for diagnosis and treatment of subacromial pain syndrome: a multidisciplinary review by the Dutch Orthopaedic Association. Acta Orthop 2014;85:314-22.

79 Eubank BH, Mohtadi NG, Lafave MR, et al. Using the modified Delphi method to establish clinical consensus for the diagnosis and treatment of patients with rotator cuff pathology. BMC Med Res Methodol 2016;16:56.

80 Oliva F, Piccirilli E, Bossa M, et al. I.S.Mu.L.T - Rotator cuff tears guidelines. Muscles Ligaments Tendons J 2015;5:227.

81 Washington State Department of Labor and Industries. Shoulder conditions: diagnosis and treatment guideline. Washington, 2013:28.

82 Bryans R, Decina P, Descarreaux M, et al. Evidence-based guidelines for the chiropractic treatment of adults with neck pain. J Manipulative Physiol Ther 2014;37:42-63.

83 State of Colorado Department of Labor and Employment: Division of Worker's Compensation. Cervical spine injury: medical treatment guidelines. Denver, Colorado, 2014:96.

84 Monticone M, lovine R, de Sena G, et al. The Italian Society of Physical and Rehabilitation Medicine (SIMFER) recommendations for neck pain. G Ital Med Lav Ergon 2013;35:36-50.

85 Hegmann K, Travis R, Andersson GB, et al. Cervical and thoracic spine disorders. Elk Grove Village, Illinois, USA: American College of Occupational and Environmental Medicine (ACOEM., 2016:1-711.

86 Barton CJ, Lack S, Hemmings S, et al. The 'Best Practice Guide to Conservative Management of Patellofemoral Pain': incorporating level 1 evidence with expert clinical reasoning. Br J Sports Med 2015;49:923-34.

87 Braddock E, Greenlee J, Hammer R, et al. Manual medicine guidelines for musculoskeletal injuries. Sonora (CA): Academy for Chiropractic Education, 2013:70.

88 State of Colorado Department of Labor and Employment: Division of Worker's Compensation. Lower extremity injury: medical treatment guidelines. Denver, Colorado, 2016:264.

89 Bussières AE, Patey AM, Francis JJ, et al. Identifying factors likely to influence compliance with diagnostic imaging guideline recommendations for spine disorders among chiropractors in North America: a focus group study using the Theoretical Domains Framework. Implement Sci 2012;7:82. 